\title{
ABKÜRZUNGEN GEOGRAPHISCHER NAMEN
}

\begin{tabular}{|c|c|c|c|}
\hline AK & Alaska & $\mathrm{NC}$ & North Carolina \\
\hline $\mathrm{AL}$ & Alabama & $\mathrm{Ndb}$. & Niederbayern \\
\hline AR & Arkansas & Nds. & Niedersachsen \\
\hline AZ & Arizona & ND & North Dakota \\
\hline Br. & Breisgau & NE & Nebraska \\
\hline Brandenb. & Brandenburg & $\mathrm{NH}$ & New Hampshire \\
\hline $\mathrm{BRD}$ & Bundesrepublik Deutschland & $\mathrm{NJ}$ & New Jersey \\
\hline Burgenl. & Burgenland & NM & New Mexico \\
\hline $\mathrm{CA}$ & Kalifornien & NV & Nevada \\
\hline $\mathrm{CO}$ & Colorado & NY & New York (nur als Staat) \\
\hline $\mathrm{CT}$ & Connecticut & Obb. & Oberbayern \\
\hline DDR & Deutsche Demokratische & Öst., öst. & Österreich, österreichisch \\
\hline & Republik & $\mathrm{OH}$ & Ohio \\
\hline DE & Delaware & OK & Oklahoma \\
\hline Dtl. & Deutschland & OR & Oregon \\
\hline Erzgeb. & Erzgebirge & $\mathrm{PA}$ & Pennsylvania \\
\hline europ. & europäisch & Pomm. & Pommern \\
\hline $\mathrm{FL}$ & Florida & Pr. & Preußen \\
\hline Frankfurt/M. & Frankfurt am Main & Rhld. & Rheinland \\
\hline Frankfurt/O. & Frankfurt (Oder) & RI & Rhode Island \\
\hline Friesl. & Friesland & Sa. & Sachsen \\
\hline GA & Georgia & Schles. & Schlesien \\
\hline $\mathrm{HI}$ & Hawaii & SC & South Carolina \\
\hline Holst. & Holstein & SD & South Dakota \\
\hline IA & Iowa & Siebenb. & Siebenbürgen \\
\hline ID & Idaho & Thür. & Thüringen \\
\hline IL & Illinois & $\mathrm{TN}$ & Tennessee \\
\hline IN & Indiana & $\mathrm{TX}$ & Texas \\
\hline KS & Kansas & UdSSR & Union der Sozialistischen \\
\hline Kurl. & Kurland & & Sowjetrepubliken \\
\hline KY & Kentucky & USA & Vereinigte Staaten von \\
\hline LA & Louisiana & & Amerika \\
\hline Livl. & Livland & UT & Utah \\
\hline MA & Massachusetts & VA & Virginia \\
\hline MD & Maryland & Vogtl. & Vogtland \\
\hline Mecklenb. & Mecklenburg & VT & Vermont \\
\hline $\mathrm{ME}$ & Maine & WA & Washington (nur als Staat) \\
\hline MI & Michigan & Westf. & Westfalen \\
\hline $\mathrm{MN}$ & Minnesota & WI & Wisconsin \\
\hline $\mathrm{MO}$ & Missouri & WV & West Virginia \\
\hline MS & Mississippi & WY & Wyoming \\
\hline MT & Montana & Württ. & Württemberg \\
\hline
\end{tabular}

